\title{
Hierarchical simulation of aquifer heterogeneity: implications of different simulation settings on solute-transport modeling
}

\author{
Alessandro Comunian ${ }^{1} \cdot$ Leonardo De Micheli $^{2}$ - Claudio Lazzati ${ }^{3}$ - Fabrizio Felletti ${ }^{1}$. \\ Francesca Giacobbo $^{2}$ - Mauro Giudici ${ }^{1,4,5} \cdot$ Riccardo Bersezio $^{1,5}$
}

\begin{abstract}
The fine-scale heterogeneity of porous media affects the large-scale transport of solutes and contaminants in groundwater and it can be reproduced by means of several geostatistical simulation tools. However, including the available geological information in these tools is often cumbersome. A hierarchical simulation procedure based on a binary tree is proposed and tested on two real-world blocks of alluvial sediments, of a few cubic meters volume, that represent smallscale aquifer analogs. The procedure is implemented using the sequential indicator simulation, but it is so general that it can be adapted to various geostatistical simulation tools, improving their capability to incorporate geological information, i.e., the sedimentological and architectural characterization of heterogeneity. When compared with a standard sequential indicator approach on bi-dimensional simulations, in terms of proportions and connectivity indicators, the proposed procedure yields reliable results, closer to the reference observations. Different ensembles of three-dimensional simulations based on different hierarchical sequences are used to perform numerical experiments of conservative solute transport and to obtain ensembles of equivalent pore velocity and dispersion coefficient at the scale length of the blocks (meter). Their
\end{abstract}

Alessandro Comunian

alessandro.comunian@unimi.it

1 Dipartimento di Scienze della Terra “A. Desio”, Università degli Studi di Milano, Milan, Italy

2 Dipartimento di Energia, Politecnico di Milano, Milan, Italy

3 Università degli Studi di Milano, Milan, Italy

4 CINFAI (Consorzio per la Fisica delle Atmosfere e delle Idrosfere), Tolentino, MC, Italy

5 CNR-IDPA (Consiglio Nazionale delle Ricerche, Istituto per la Dinamica dei Processi Ambientali), Milan, Italy statistics are used to estimate the impact of the variability of the transport properties of the simulated blocks on contaminant transport modeled on bigger domains (hectometer). This is investigated with a one-dimensional transport modeling based on the Kolmogorov-Dmitriev theory of branching stochastic processes. Applying the proposed approach with diverse binary trees and different simulation settings provides a great flexibility, which is revealed by the differences in the breakthrough curves.

Keywords Geostatistics · Scaling · Alluvial aquifers · Kolmogorov-Dmitriev $\cdot$ Solute transport

\section{Introduction}

The difficulty to observe, map and model the heterogeneity and the spatial variability of the physical properties of geological media at fine scale, relevant to forecast the fate of solutes and contaminants in groundwater at long times and large distances, favored the development of geostatistical and stochastic tools. Several authors, including Klingbeil et al. (1999) and Anderson (1989), proposed the regrouping of the rock types in a relatively small number of classes, called hydrofacies, characterized by similar hydrodynamic and hydrodispersive properties. The spatial distribution of hydrofacies, coded through a categorical variable (indicator), is obtained by stratigraphic modelling followed by stochastic simulation, possibly conditioned via field data. Then, the spatial distribution of physical quantities (e.g., hydraulic conductivity) is approximated by associating the corresponding estimates of the physical characteristic parameters to the regions occupied by each hydrofacies. The resulting spatial distributions of hydrodynamic and hydrodispersive properties can be used to simulate groundwater flow and solute transport in 
response to different natural conditions, exploitation scenarios or contamination episodes, and are useful in a range of practical applications, including, for instance, environmental impact assessment and risk analysis of waste disposal sites, as well as design of remediation activities.

The need to constrain the simulation framework to the geological information on architecture of aquifers and to the depositional settings has been pushing research in the direction of hierarchical approaches. Several authors proposed the improvement of the stochastic simulation by mapping the principal discontinuities with interpolation of field data and then by separately reconstructing distribution of the hydrofacies inside each single geological body with diverse simulation methods. Jussel et al. (1994), for example, reconstructed gravel deposits first by subdividing the domain in individual sedimentary elements, then by simulating, inside each element, objects with a shape resulting from the statistical properties of field data. Weissmann and Fogg (1999) applied a similar approach, by interpolating at first the main stratigraphic sequences and then using transition-probabilities Markov chains methods (Carle 1996) to simulate the heterogeneity inside each sequence. In a similar fashion, Comunian et al. (2011) applied ordinary kriging to reproduce the shape of the largescale sedimentary units and then multiple-point statistics (Guardiano and Srivastava 1993; Strebelle 2002) to reproduce the small-scale heterogeneity within each sedimentary unit. In all these works, the simulation workflow contains at least the following two steps: first, the shape of high-rank sedimentary units is simulated with a well-known interpolation technique; second, their internal architecture is simulated with stochastic methods. These approaches allow the simulation of complex and nested heterogeneities with a good degree of realism; however, in some cases, their application can be burdensome because it requires the use of different methods and tools in sequence.

In this paper, an alternative hierarchical procedure aimed at simplifying the simulation workflow is developed, as a refinement of the approach already proposed by Zappa et al. (2006). The basic idea is the subdivision of the stochastic simulation according to a binary tree: at each node of the binary tree, two hydrofacies or groups of hydrofacies are simulated against each other, whereas at the end, the simulations are merged to obtain the complete and final spatial distribution of hydrofacies. In so doing, the first interpolation step normally required by other hierarchical simulation procedures is bypassed, and the hierarchical architecture is integrated in a more direct fashion into the procedure. The hierarchical simulation procedure proposed here is general and can be implemented using different geostatistical simulation techniques, including transition-probability Markov chain (Carle 1996), multiple-point statistics (Guardiano and Srivastava 1993; Strebelle 2002) or truncated pluri-Gaussian simulations (Le Loc'h et al. 1994). In particular, in the application presented in this work, the procedure is implemented using the sequential indicator simulation (SISIM) of Deutsch and Journel (1997), and is referred to as hierarchical sequential indicator simulation (HSISIM) as proposed by Zappa et al. (2006). SISIM is just one of the possible algorithms that can be implemented in the proposed procedure and was selected because, despite its important limitations (Emery 2004), it remains one of the most used and well-known facies simulation methods. The results of the application of HSISIM are compared here with those of a standard non-hierarchical approach, i.e., plain SISIM procedure with one variogram model for each hydrofacies.

Other authors have proposed hierarchical simulation procedures that include SISIM. Seifert and Jensen (1999), for example, demonstrated the improvements obtained using SISIM within multibinary models; however, their tests did not include connectivity indicators. Compared to the Seifert and Jensen approach, the multibinary procedure proposed here follows a more complete hierarchical workflow. Cherubini et al. (2009) introduced a nested simulation method that uses SISIM, in which the hydrofacies were grouped as lithotype sets and simulation constraints based on the vertical marginal proportions and on transition probability matrices were also included. In the present work, such constraints are not taken into account explicitly, and the complexity of the geological heterogeneity is incorporated directly by simulating each facies separately according to a given binary tree. Although the approach proposed here is a refinement of the one proposed by Zappa et al. (2006), in this work, a number of new improvements, simulations, comparisons and tests are made: (1) the HSISIM procedure is completely automated and refined; (2) a 2D validation against real world data is performed; (3) a thorough comparison is made with the standard SISIM approach, based on visual inspection, facies proportions, connectivity indicators and three-dimensional (3D) transport simulations (particle tracking); (4) the sensitivity to the choice of different simulation settings and different binary trees is investigated on one-dimensional (1D) domains about $100 \mathrm{~m}$ long by transport simulations performed with the Kolmogorov-Dmitriev (KD) approach. The aforementioned novelties with respect to previous related research now permit the systematic use of ensembles of multiple realizations to analyze, in a Monte Carlo framework, the variability of the stochastic simulations and its effects on flow and transport modeling.

Therefore, the objective of this paper is to propose a hierarchical procedure which is fully based on a single simulation technique, and to test it with field data sets at the fine scale (resolution of $2 \mathrm{~cm}$ ) with a two-fold approach. First, the proposed procedure is compared with a standard non-hierarchical approach by means of visual inspection of the results, analysis of facies proportions and connectivity indicators, and modeling transport with particle tracking on 3D domains of the same 
size of the blocks. Second, with the aforementioned 3D particle-tracking approach, estimates of equivalent longitudinal dispersion coefficient $D_{1}$ and equivalent average porewater velocity $v$ are obtained for each realization; then, the statistics of $D_{1}$ and $v$ are used to investigate the sensitivity of the proposed procedure to different choices of simulation settings and binary trees by means of 1D transport simulations performed with the KD approach on about 100-m-long domains.

The model blocks that compose the data set are described; then the methods are illustrated, including details about the hierarchical simulation procedure and the flow and transport experiments. The hierarchical procedure is compared with a standard nonhierarchical procedure and validated on a twodimensional (2D) test case. The results obtained using different parameter settings and different binary trees are illustrated by means of $1 \mathrm{D}$ transport simulations performed with the KD approach. Finally, there are remarks on the results and on the procedure.

\section{The case study}

The data set used to test the proposed simulation procedure derives from a thorough sedimentological study performed by Zappa et al. (2006) on three blocks of glacio-fluvial sediments, which were dug in an open quarry in the Ticino Valley, northern Italy (Fig. 1).For the sake of simplicity, this study considers only two of the three model blocks examined by Zappa et al. (2006), namely MB2 and MB3. In fact, MB2 and MB3 already represent relatively diverse heterogeneity patterns: MB2 (Fig. 2b) was dug into a braided river gravel bar and consisted of a high-conductivity and anisotropic facies association of sandy gravel and open framework gravel (coarse-grained bedload sheet) with a minor proportion of coarse and fine-laminated sand (upper flow regime lowrelief bedform); MB3 (Fig. 2c) constitutes a less conductive and less anisotropic facies association than MB2, consisting of poorly sorted sandy gravels that form a mostly disorganized massive deposit with some trough-laminated sand lenses and abundant sparse pebbles.

This data set is chosen because it had already been the subject of a deep investigation of several works and approaches (Baratelli et al. 2011, 2014; Zappa et al. 2006; Giudici et al. 2012; Vassena et al. 2010). While the reader is referred to Zappa et al. (2006) for a thorough description of the geological and sedimentological framework, here the relevant details for the specific goals of this paper are briefly recalled.

For each MB, a great portion of the lateral faces was analyzed from the sedimentological point of view and the facies analysis yielded maps of the exposed faces over a regular discretization grid with 2-cm spacing (Fig. 2). The facies were then grouped according to their lithological, textural and hydraulic characteristics in a set of operative facies (six for and MB2, seven for MB3, Table 1). Each operative facies was characterized by a range of values of porosity and an average value of hydraulic conductivity $K$ estimated from the integration of laboratory measurements on undisturbed samples for the fine grained facies, estimates from Hazen's and KozenyKarman's empirical formulas (Vukovic and Soro 1992) and literature data, as discussed by Zappa et al. (2006). In the present work, for the sake of simplicity, the porosity is considered constant and equal to 0.4 for all the facies.

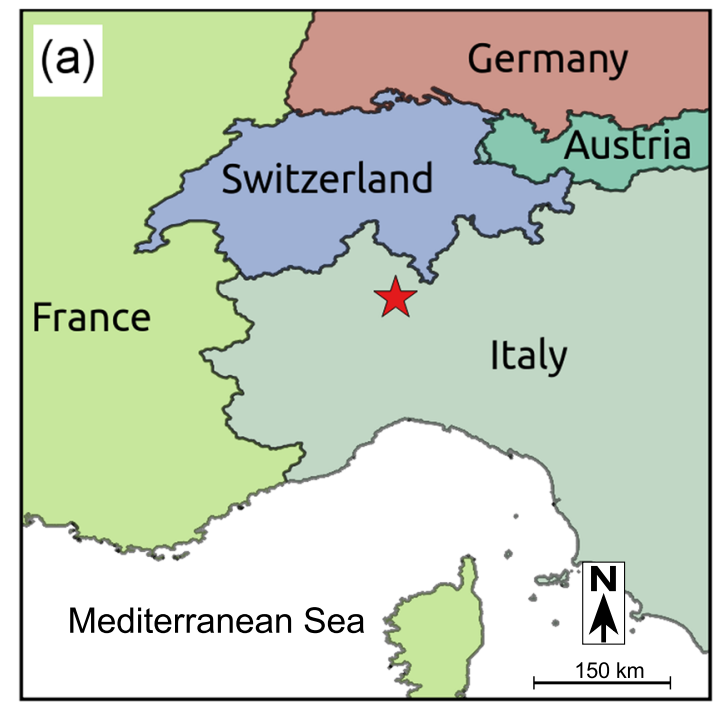

Fig. 1 Location and geological setting of the gravel pit where the model blocks were dug: a location, Ticino Valley, northern Italy (star); b geological setting (after Bersezio et al. 1999): Go: Middle Pleistocene glacial sediments (Golasecca Alloformation); $B s u, B M o, B D a, B C a$ :

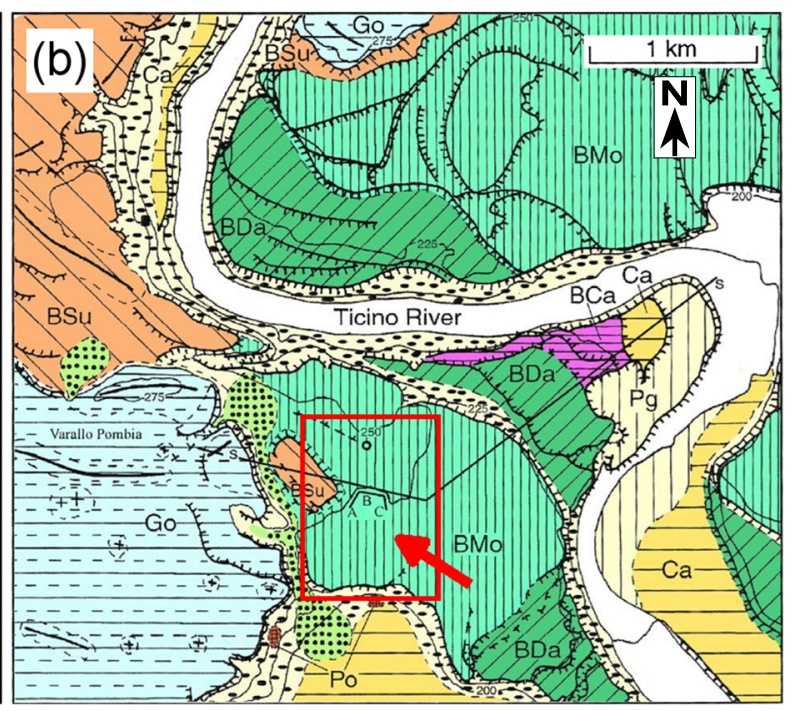

glacio-fluvial terraces of middle-late Pleistocene age (Besnate Allogroup), Ca: glacio-fluvial sediments of the Last Glacial Maximum (Cantù Alloformation); Pg: post-glacial sediments 
Fig. 2 The data set used to reconstruct $\mathrm{MB} 2$ and $\mathrm{MB} 3$ comes from the interpretation of photomosaics. Each square cell of the data set has 2-cm sides (vertical exaggeration of 3D pictures of the model blocks is $2 \times)$. a One of the photomosaic faces used to build up the dataset (here face $A, M B 2$ ), b MB2 data (strong horizontal anisotropy, with high conductive hydrofacies), c MB3 data (almost isotropic, hydraulic conductivities lower than MB2) (a) MB2, photomosaic face A
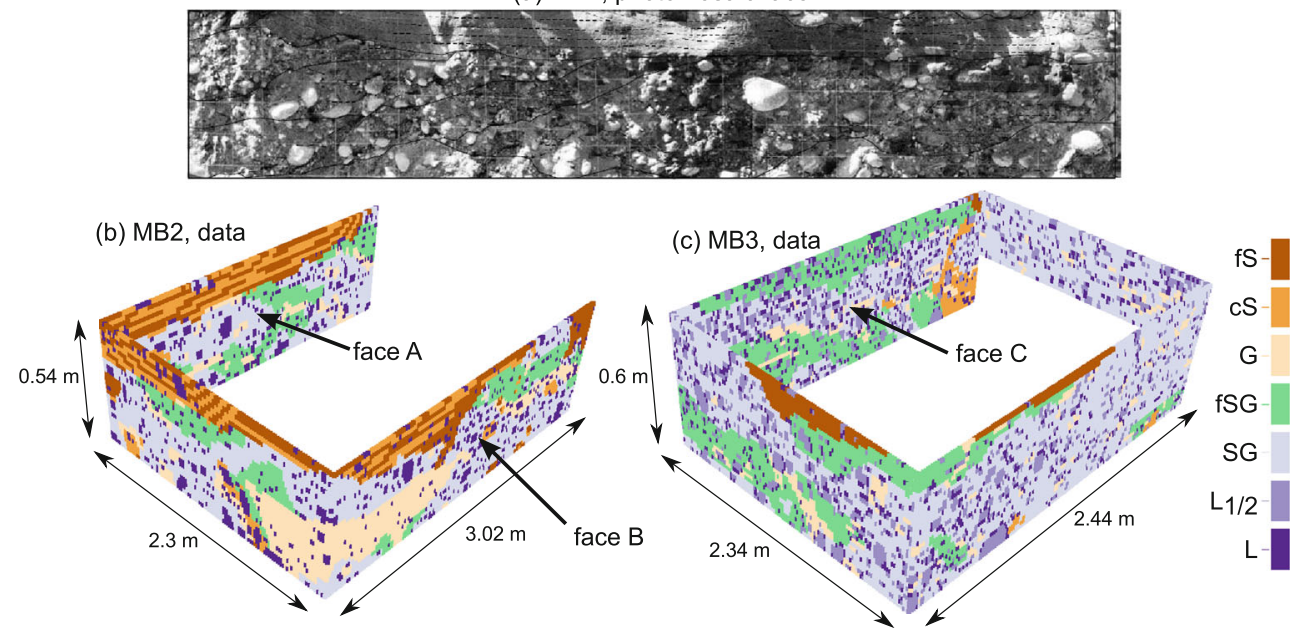

The maps of operative facies for the lateral faces of the MBs (Fig. 2) are the input data for stochastic simulations of the $2 \mathrm{D}$ and $3 \mathrm{D}$ distribution of operative facies in the entire volume of each MB; these are used for variographic analysis (computing the experimental indicator variograms and fitting model variograms), for the computation of facies proportions, and as conditioning data for the stochastic simulations.

\section{Methods}

The hierarchical simulation procedure proposed in this work and the transport simulation workflow performed to apply the Kolmorogov-Dmitriev approach are summarized in Figs. 3 and 4 respectively.The starting point of the hierarchical simulation procedure is the field data, which in this study consists of two blocks of alluvial sediments with a volume between 3 and $4 \mathrm{~m}^{3}$ described in the previous section (Fig. 2). Here the hierarchy of stratigraphic units proposed by Zappa et al. (2006) is adopted to define the hierarchical simulation binary tree and to split the data set into sub-sets, which correspond to an aggregation of one or more hydrofacies. The sub-sets are used to compute the relative hydrofacies proportions, for the

Table 1 Operative facies: codes, description and hydraulic conductivity. Refer to Zappa et al. (2006) for details about the geological setting and the hydraulic properties of the case study

\begin{tabular}{lll}
\hline Code & Description & $K\left[\mathrm{~m} \mathrm{~s}^{-1}\right]$ \\
\hline $\mathrm{fS}$ & Fine sand & $10^{-4}$ \\
$\mathrm{cS}$ & Coarse to medium sand & $5 \times 10^{-4}$ \\
$\mathrm{SG}$ & Sandy gravel (poorly sorted) & $6 \times 10^{-4}$ \\
$\mathrm{fSG}$ & Fine sandy gravel (well sorted) & $2 \times 10^{-3}$ \\
$\mathrm{G}$ & Open framework gravel & $5 \times 10^{-2}$ \\
$\mathrm{~L}$ & Pebbles and cobbles & $10 \times 10^{-20}$ \\
$\mathrm{~L}_{1 / 2}$ & Pebbles and cobbles occupying less than one cell & $2.8 \times 10^{-5}$ \\
\hline
\end{tabular}

variographic analysis, and to condition the simulations. The simulations are performed first in two dimensions, to allow a

\section{Hierarchical simulation procedure}

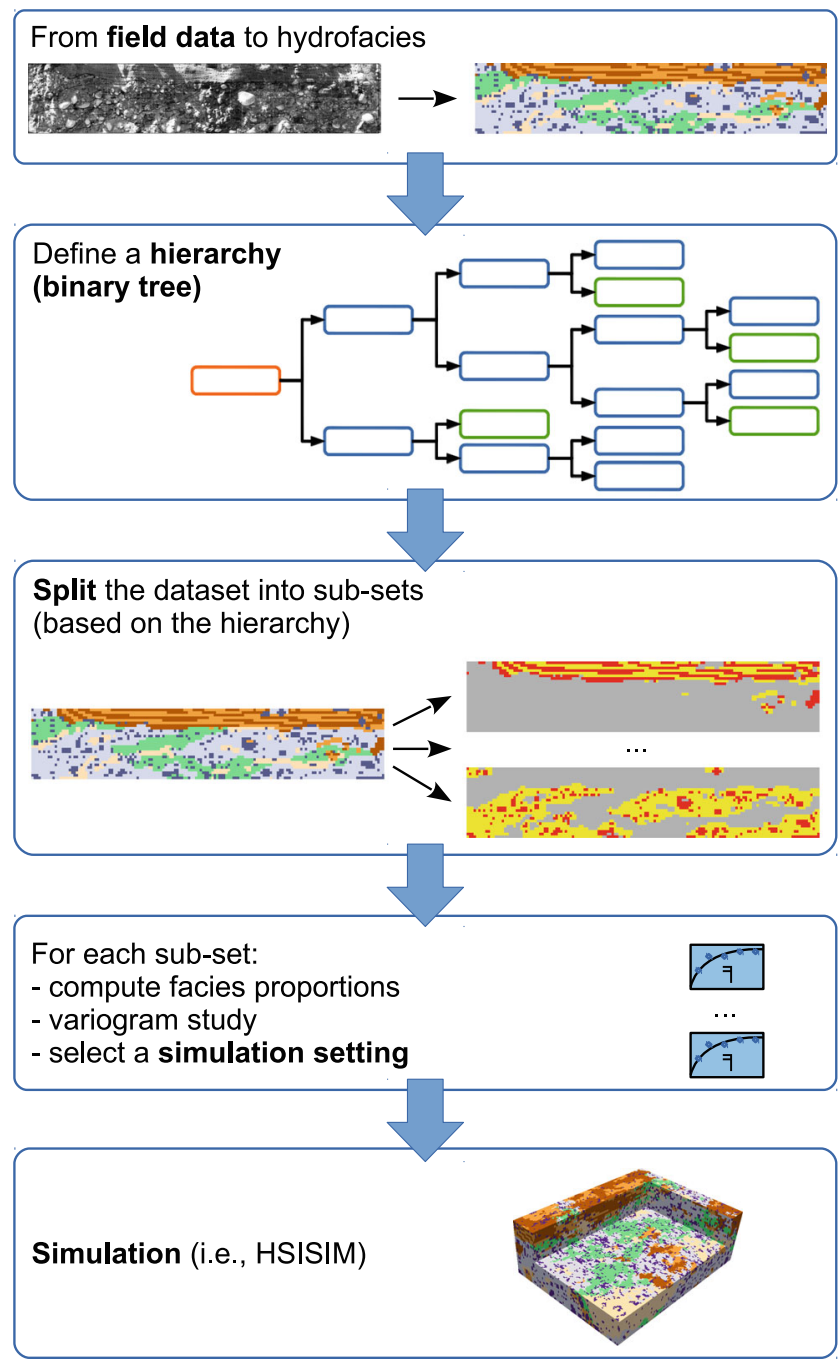

Fig. 3 Scheme of the proposed hierarchical simulation procedure 
Fig. 4 Workflow needed for the transport simulation with the Kolmogorov-Dmitriev approach. References to time are in seconds
Transport simulation workflow

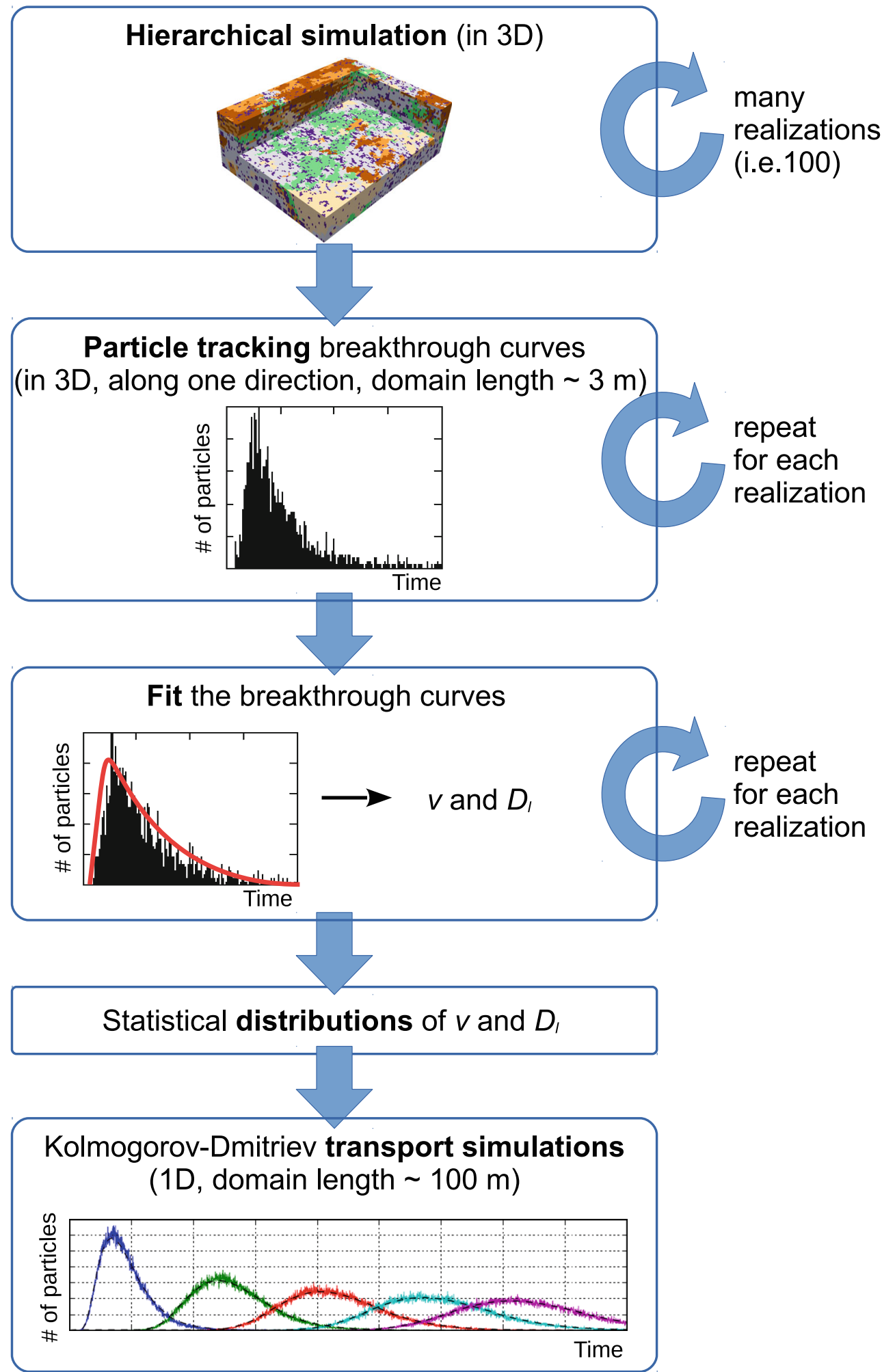

direct comparison with the field data, and then in three dimensions, taking into account for the spatial correlation provided by the 2D outcrops mapped along perpendicular directions (Fig. 2); in both 2D and 3D cases, ensembles of 100 equiprobable realizations are generated and analyzed. The results are first examined visually and with the support of connectivity indicators, which are useful to describe the heterogeneity pattern of each ensemble. 
The transport simulation workflow (Fig. 4) is based on the multiple realizations obtained with the proposed hierarchical simulation procedure. First, 3D flow (finite differences) and advective transport (particle tracking) numerical models are applied to each realization, neglecting molecular diffusion and dispersion originated by the heterogeneity of the velocity field at the pore scale. Then, the curves resulting from the particle tracking are fitted with the analytical solution of the 1D advective-dispersive equation with a least-squares criterion, obtaining probability distributions of equivalent porewater velocity $v$ and of equivalent longitudinal dispersion $D_{1}$. Then, the obtained probability distributions of $D_{1}$ and $v$ are used to simulate the transport of conservative solutes with 1D stochastic transport models based on the application of the KD theory of branching stochastic processes (Kolmogorov and Dmitriev 1947) in a Monte Carlo framework. These 1D simulations are performed on domains with a linear dimension of about $100 \mathrm{~m}$, i.e., two orders of magnitude greater that the sides of the $3 \mathrm{D}$ blocks.

\section{Hierarchical simulation procedure}

In this work, the hierarchical simulation procedure is implemented in an ad hoc computer program, named HSISIM and based on the GSLIB libraries (Deutsch and Journel 1997). The simulation procedure is illustrated in Fig. 3. First, field data are interpreted in terms of hydrofacies. Then, the basic idea is to create a hierarchical binary tree and to split the data-set accordingly. Each node in the binary tree corresponds with a SISIM simulation performed on a domain characterized by two possible categories only. Such categories could correspond to a single hydrofacies or to a collection of hydrofacies. The repeated application of SISIM permits the separation of different subcategories inside each category simulated in the parent node. The procedure is repeated until each individual hydrofacies is simulated. In this way, the simulation takes into account the geological nesting of hydrofacies, which corresponds to the sedimentological and genetic concept of facies association. Figure 5 complements the workflow illustrated in Fig. 3, and in particular the 2nd and the 3rd step of the workflow. The binary tree built upon the textural properties proposed in the detailed analysis by Zappa et al. (2006) accounts for the nesting of the litho-textural classes within the hierarchically arranged sedimentary units. The nesting of each litho-textural category within sedimentary units of different rank is illustrated in terms of facies codes (Fig. 5a) and in terms of the corresponding simplified sections (Fig. 5c). Such a procedure is necessary because the hierarchically arranged sedimentary units (facies, facies associations or architectural elements, groups of facies associations or depositional elements and so on, in increasing hierarchical ranking) are composed of different proportions of the litho-textural categories (that do not coincide with the sedimentary facies). Hence, grouping the litho-textural classes and then splitting them into sub-sets for simulation, permits the reproduction of the hierarchic depositional arrangement according to the relative abundance of the litho-textural classes within the sedimentary units of increasing rank.If conditioning data are available, they are grouped according to the hierarchy defined by the binary tree and used at each step to condition the simulation.

Each simulation node of HSISIM requires the assignment of three codes: $S, A$ and $B$, where $S$ is the code of the category which was simulated at one of the parent nodes and which is going to be subdivided at the examined step in two subcategories, denoted by the codes $A$ and $B$. In other words, at each node, SISIM populates the sub-domain corresponding to the category $S$ with one of the two subcategories $A$ or $B$. At the first node, $S$ occupies the whole domain under study.

Notice that only one indicator variogram is necessary for each simulation node. In fact, if $I_{\mathrm{A}}$ and $I_{\mathrm{B}}$ denote the indicator functions of the two categories of a binary domain, then $I_{\mathrm{B}}(x)=1-I_{\mathrm{A}}(x)$ at every point $x$, so that, under the intrinsic hypothesis (Isaaks and Srivastava 1989), the following equality between the two indicator variograms $\gamma_{\mathrm{A}}$ and $\gamma_{\mathrm{B}}$ holds

$$
\begin{aligned}
\gamma_{\mathrm{A}}(\mathbf{h}) & =E\left\{\left[I_{\mathrm{A}}(x)-I_{\mathrm{A}}(x+\mathbf{h})\right]^{2}\right\} \\
& =E\left\{\left[I_{\mathrm{B}}(x)-I_{\mathrm{B}}(x+\mathbf{h})\right]^{2}\right\}=\gamma_{\mathrm{B}}(\mathbf{h}) .
\end{aligned}
$$

where $E[\cdot]$ represents the expectation value of the quantity in brackets and $\mathbf{h}$ is a lag distance vector.

Therefore, HSISIM needs an indicator variogram for each node of the binary tree, whereas SISIM needs an indicator variogram for each considered hydrofacies. The number of individual simulations performed by HSISIM is greater than the number of hydrofacies and, therefore, the use of HSISIM requires some more work than SISIM to prepare the input data. Moreover, notice that the input data-files for HSISIM have the same structure as those used for SISIM. Only one additional file, which includes the number of simulation nodes and for each the three codes $A, B$ and $S$, is necessary.

In addition, the application of SISIM and HSISIM requires the marginal probability of each facies. While for SISIM, variograms and marginal probabilities are computed on the whole simulation domain, for HSISIM they are computed on each sub-domain defined by the hierarchical binary tree.

The proposed procedure is compared with the standard SISIM approach in two and three dimensions considering an ensemble of 100 equiprobable realizations for each simulation setting, obtained by changing the random seed. A visual inspection of some realizations and a quantitative analysis of facies proportions and connectivity are performed to compare the simulations of SISIM and HSISIM.

In particular, connectivity is quantified with the normal and the intrinsic connectivity defined as follows (Vassena et al. 2010): let $\Omega$ be the domain under study and let $\Omega_{f}$ be the 


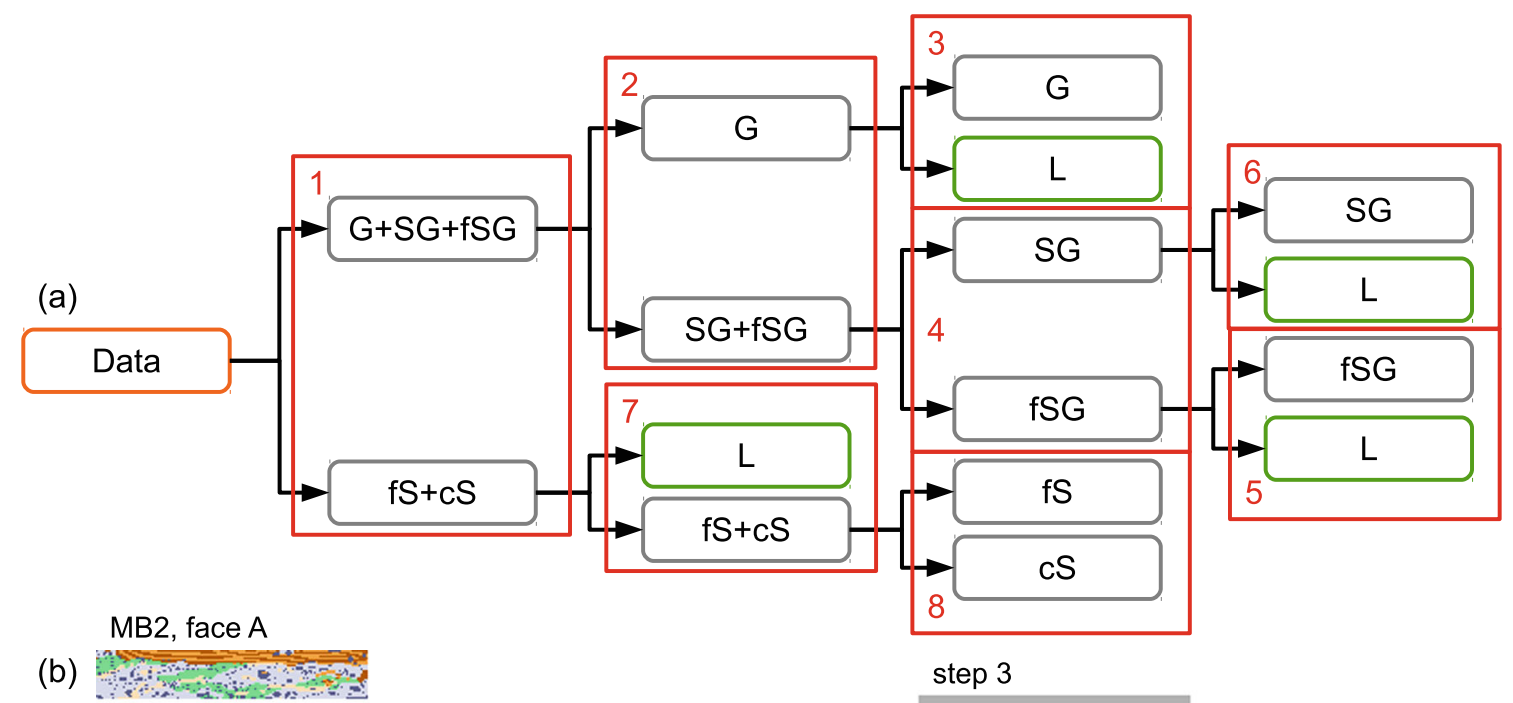

(b)

(c)

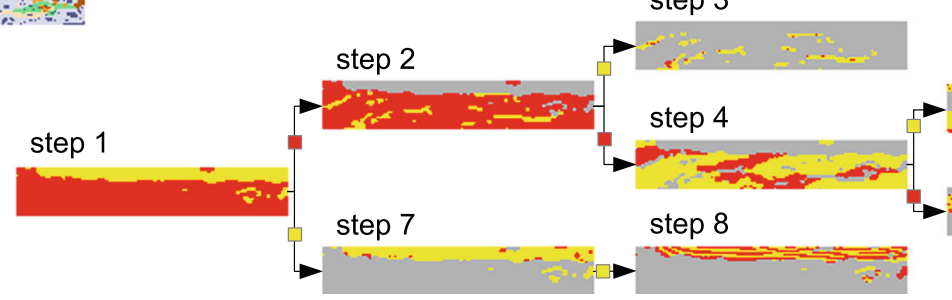

Fig. 5 a Flowchart of the binary tree based on the established textural hierarchy and used to simulate MB2. Note that cobbles (facies $L$, green boxes) are simulated separately within the main facies to take into account their different spatial variability. The numbers in the red boxes correspond

to the ID of the variogram used to model the two enclosed groups of facies (Table 3). b Data set used to illustrate the hierarchical procedure. c The hierarchical procedure applied to the data set shown in $\mathbf{b}$

collection of points occupied by the facies $f$. Two points $x \in \Omega_{f}$ and $y \in \Omega_{f}$ are said to be connected - denoted as $x \leftrightarrow y$-if there is a continuous path joining the two points and fully included in $\Omega_{f}$. Then, the total connectivity can be defined with the formalism of probability theory in terms of probability $P$ as

$C_{f}^{t}=P\left[x \leftrightarrow y, \quad x \in \Omega_{f}, \quad y \in \Omega_{f} \mid x \neq y\right]$

and the intrinsic connectivity as

$C_{f}^{*}=P\left[x \leftrightarrow y \mid x \in \Omega_{f}, y \in \Omega_{f}, x \neq y\right]$.

The intrinsic connectivity $C_{f}^{*}$ is high for hydrofacies that form pixel/voxel clusters and low if hydrofacies are dispersed. Diversely, the total connectivity $C_{f}^{t}$ is also influenced by the abundance of the hydrofacies. Connectivity indicators can be considered as proxies of the hydraulic connection; their physical meaning is thoroughly described in Vassena et al. (2010). Note that other definitions of connectivity indicators were proposed in literature; see for example the review by Renard and Allard (2013).

\section{Workflow for the Kolmogorov-Dmitriev transport modeling}

Figure 4 illustrates the workflow implemented to perform the transport modeling with the Kolmogorov-Dimitriev approach. The starting point is the many realizations produced by a simulation procedure (Fig. 4, first step). First, 3D particle-tracking numerical experiments are performed for the 3D model blocks on the two ensembles of 100 simulations each (Fig. 4, second step). In particular, an approach similar to that applied by Baratelli et al. (2011), Dell'Arciprete et al. (2014), and Vassena et al. (2010) was used to compute both equivalent hydraulic conductivity, $v$ and $D_{1}$ with the computer program mg_up3D (Zappa et al. 2006). This computer program is based on a standard conservative finite-differences discrete model of saturated groundwater flow and is specifically designed to perform well for situations with high contrasts of hydraulic conductivity, such as for the test cases of this paper, which include impermeable cells. Advective transport of conservative solutes is modeled with a particle tracking method.

The values of $D_{1}$ and $v$ are obtained for each realization through the following procedure corresponding to the second and third steps of the workflow shown in Fig. 4: (1) the head field is simulated for an average 1D stationary flow, corresponding to a unit hydraulic gradient along a given Cartesian axis (the $x$ axis in this case) by prescribing constant Dirichlet boundary conditions along the two lateral faces perpendicular to the average flow direction and no flow along the remaining boundary faces; (2) an instantaneous injection of solute is simulated through the inflow boundary face, by 
injecting contaminant particles proportionally to the flow rate; (3) each particle is traced along the model block by applying a trilinear interpolation of the velocity field and a fourth-order Runge-Kutta scheme for the integration of the equation of motion (Anderson and Woessner 1991); (4) the times at which the particles cross some control planes are stored and successively used to compute the breakthrough curve (BTC) at the end of the model block; (5) the cumulative BTC is fitted with the analytical solution of the transport equation for transport in a stationary and homogeneous 1D flow in a semi-infinite medium, so that the equivalent pore water velocity and longitudinal dispersion coefficient are obtained. The analytical solution for the flux-averaged concentration $C_{\mathrm{F}}$ used to fit the BTCs (Kreft and Zuber 1978; Hubert 1970) is

$C_{F}(x, t)=\frac{M}{v} \frac{x}{\sqrt{4 \pi D_{1} t^{3}}} \exp \left[-\frac{(x-v t)^{2}}{4 D_{1} t}\right]$

where $M$ denotes the mass of solute, per unit surface, instantaneously injected at the time $t=0$. At the fourth step of the simulation workflow illustrated in Fig. 4, the results of the aforementioned flow and transport simulations are used to obtain frequency distribution curves for $v$ and $D_{1}$. The obtained distributions are fitted with appropriate probability density functions (pdfs), whose parameters are used to model 1D transport of contaminants by means of a Monte Carlo approach based on the KD theory of branching stochastic processes (Fig. 4, last step). In the KD theory, the contaminant particles movement is described through transition rates that depend on the hydrodispersive parameters of the medium and the average pore-water velocity (Marseguerra and Zio 1997; Giacobbo and Patelli 2007). Such transition rates are determined step-by-step for each contaminant particle by sampling $v$ and $D_{1}$ from the aforementioned pdfs. The flexibility and the computational efficiency of this approach allows for performing transport simulations on domains extended to lengths two orders of magnitude greater than those of the model blocks, namely about $100 \mathrm{~m}$. In the implementation of the KD approach proposed by Marseguerra and Zio (1997) and Giacobbo and Patelli (2007), the computed concentrations are obtained in terms of resident (volumeaveraged) concentration $C_{\mathrm{R}}$ (Kreft and Zuber 1978). Therefore, the resulting BTC obtained with the KD approach can be compared with the analytical expression of $C_{\mathrm{R}}$ obtained setting the median values in the following expression

$C_{\mathrm{R}}(x, t)=\frac{M}{n} \frac{x}{\sqrt{4 \pi \tilde{D}_{1} t}} \exp \left[-\frac{(x-\tilde{v} t)^{2}}{4 \tilde{D}_{1} t}\right]$

were $\tilde{v}$ and $\tilde{D}_{1}$ are the median values of $v$ and $D_{1}$ respectively.

In the following sections, the results obtained with the $\mathrm{KD}$ approach will be used to study the sensitivity of the hierarchical approach to the choice of the binary tree and to different simulation settings.

\section{Results}

\section{HSISIM vs SISIM}

The comparison between the proposed hierarchical procedure and the standard SISIM is carried out first in 2D and then in $3 \mathrm{D}$. The $2 \mathrm{D}$ comparisons include a validation compared to field data and are based on visual inspection, facies proportions and connectivity indicators; the 3D comparisons are also based on particle-tracking simulations.

\section{$2 D$ validation}

Prior to its application in 3D, the simulation procedure is validated on two 2D case studies, one for each considered block, where it is possible to compare the results with the observed 2D outcrops. First, one 2D outcrop for each model block is selected (Figs. 2 and 6a). From each outcrop, three vertical columns of data are extracted and considered as conditioning data for the simulation (Fig. 6, red boxes). The selected 2D outcrops are used in their entirety to compute the facies proportions and the variogram models used for the SISIM and the HSISIM simulation, the latter being performed considering the stratigraphic hierarchy shown in Fig. 5.

The results can be directly compared with the observed outcrops, based on a number of criteria. They are first compared visually (Fig. 6), then in terms of the reproduction of the hydrofacies proportions, the intrinsic and the total connectivity on a total of 100 realizations for each simulation method.

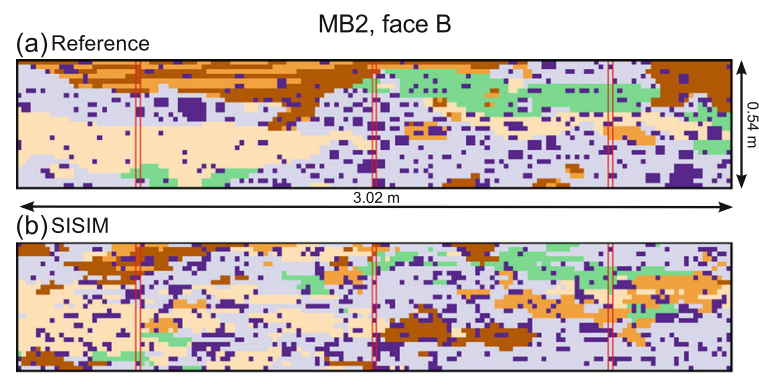

(c) HSISIM

fS
cS
G
fSG
$S G$
$L_{1 / 2}$

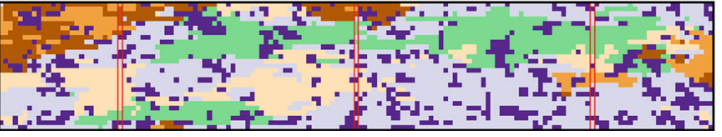

Fig. 6 2D visual validation for MB2: a reference face used to compute the variograms, the proportions and the reference connectivity indicators; b one of the 100 equiprobable realizations obtained with the standard SISIM approach; c one of the 100 equiprobable realizations obtained with the proposed hierarchical procedure. The long red boxes indicate the positions of the vertical wells extracted from the reference and considered as conditioning data in the SISIM and HSISIM simulations 
Overall, the external shape of the reference sedimentary units (Fig. 6a) is slightly better reproduced by the hierarchical procedure (Fig. 6c) rather than SISIM (Fig. 6b). Similar considerations can be made for the horizontal and vertical connectivity of a number of textural classes, as well as for the association of the textural classes within the depositional units (see for instance the S-G association at the top left of Fig. 6a,c and the $\mathrm{fSG}-\mathrm{SG}$ association throughout).

A root mean square error (RMSE) is computed for the ensemble of 100 realization for each hydrofacies and for the facies proportions and the connectivity indicators (Table 2). Here, for the sake of brevity, only the results concerning MB2 are reported but overall, for both MB2 and MB3, the RMSE errors are smaller for the HSISIM simulations than for the standard SISIM approach.

\section{D Hydrofacies simulations}

In this subsection, SISIM and HSISIM are compared in 3D using multiple realizations of target simulation domains of size $151 \times 115 \times 27$ and $117 \times 122 \times 30$ cells for MB2 and MB3 respectively. For the application of HSISIM, the same textural hierarchies used in the 2D validation are adopted. Figure 5 represents the binary tree based on the established textural hierarchy used to simulate MB2, with the corresponding variogram models reported in Table 3. Note that, for the first nodes of the binary tree, cobbles (facies L, green boxes in Fig. 5a) are included in the main facies in which they belong. They are simulated explicitly in the daughter nodes to take into account their different spatial structure in every distinct geological unit.

Since a visual comparison of all the 100 SISIM realizations and the 100 HSISIM realizations is not feasible, only one realization for each simulation method is selected randomly and shown in Fig. 7 for the visual comparison. Overall, the differences between the two realizations are not striking; however, some remarks can be made that distinguish the two realizations. First, the size and distribution of cobbles and pebbles (facies L) appears to be more realistic and in accordance with the outcrop observation in the HSISIM realizations.
Table 3 Variogram models used to simulate MB2 with HSISIM (nugget $=0$ )

\begin{tabular}{llllll}
\hline ID & Facies groups & & Model & Ranges $(x, y, z)[\mathrm{m}]$ & Sill [-] \\
\hline 1 & (G, SG, fSG) & (fS, cS) & Exponential & $(0.56,0.56,0.10)$ & 0.10 \\
2 & $(\mathrm{G})$ & $(\mathrm{fSG}, \mathrm{SG})$ & Exponential & $(0.32,0.32,0.08)$ & 0.12 \\
3 & $(\mathrm{G})$ & $(\mathrm{L})$ & Exponential & $(0.06,0.07,0.02)$ & 0.10 \\
4 & $(\mathrm{fSG})$ & $(\mathrm{SG})$ & Spherical & $(0.44,0.52,0.10)$ & 0.18 \\
5 & $(\mathrm{fSG})$ & $(\mathrm{L})$ & Exponential & $(0.04,0.05,0.01)$ & 0.12 \\
6 & $(\mathrm{SG})$ & $(\mathrm{L})$ & Exponential & $(0.07,0.04,0.02)$ & 0.15 \\
7 & $(\mathrm{fS}, \mathrm{cS})$ & $(\mathrm{L})$ & Exponential & $(0.04,0.08,0.02)$ & 0.07 \\
8 & $(\mathrm{fS})$ & $(\mathrm{cS})$ & Spherical & $(0.12,0.22,0.02)$ & 0.23 \\
\hline
\end{tabular}

Acting on the variogram parameters, one would probably obtain a better representation for both SISIM and HSISIM, yet for this study, it was decided to avoid iterative steps back-andforth from the variogram model and the simulation to keep the comparison as objective as possible. Another important difference between the two realizations is the layering of the sand structures (fS and cS) that can be observed on the top of the domain. Here the layering observed in the outcrops is better preserved in the HSISIM simulations, both considering the external shape/lateral continuity and the internal architecture of the sedimentary units. This kind of observation holds also for the open-framework gravel bodies $(\mathrm{G})$ that are realistically clustered into concave-up lens-shaped units fringed by wellsorted fSG margins and include a limited amount of oversized gravel pebbles (L). Another interesting improvement of the hierarchic simulations is the realistic continuity of the sand layers that separate the SG framework from the G lenses.

This is a very typical feature of the alluvial deposits where MB2 was dug that strongly influences preferential flow paths: openwork gravel lenses $(\mathrm{G})$ cut into a sandy gravel framework (SG) that progressively fines upwards and laterally into sand and very fine sand (cS and fS). All of these features were captured better by HSISIM than by SISIM (Fig. 7). As a very broad and qualitative comment, it can be observed that the geological descriptive information about heterogeneity and anisotropy of the MB2 hydrofacies associations is better accounted by the hierarchic approach than by the non-
Table 2 RMSE for the 100 2D simulations performed for the MB2. An asterisk is added to facilitate the recognition of smaller errors

\begin{tabular}{|c|c|c|c|c|c|c|}
\hline \multirow[t]{2}{*}{ Facies } & \multicolumn{2}{|l|}{ Proportions } & \multicolumn{2}{|c|}{ Intrinsic connectivity } & \multicolumn{2}{|c|}{ Total connectivity } \\
\hline & SISIM & HSISIM & SISIM & HSISIM & SISIM & HSISIM \\
\hline fS & $2.25 \times 10^{-3}$ & $* 1.66 \times 10^{-3}$ & $* 1.10 \times 10^{-2}$ & $1.38 \times 10^{-2}$ & $6.08 \times 10^{-6}$ & $* 5.51 \times 10^{-6}$ \\
\hline $\mathrm{cS}$ & $1.01 \times 10^{-3}$ & $* 7.16 \times 10^{-4}$ & $1.74 \times 10^{-2}$ & $* 9.86 \times 10^{-3}$ & $4.19 \times 10^{-6}$ & $* 6.99 \times 10^{-7}$ \\
\hline G & $4.11 \times 10^{-3}$ & $* 2.70 \times 10^{-3}$ & $* 9.68 \times 10^{-2}$ & $1.06 \times 10^{-1}$ & $2.83 \times 10^{-4}$ & $* 2.42 \times 10^{-4}$ \\
\hline fSG & $* 1.26 \times 10^{-3}$ & $1.78 \times 10^{-3}$ & $1.10 \times 10^{-1}$ & $* 1.02 \times 10^{-1}$ & $1.41 \times 10^{-5}$ & $* 1.39 \times 10^{-5}$ \\
\hline SG & $2.47 \times 10^{-3}$ & $* 2.42 \times 10^{-3}$ & $5.71 \times 10^{-2}$ & $* 4.70 \times 10^{-2}$ & $1.10 \times 10^{-3}$ & $* 9.24 \times 10^{-4}$ \\
\hline $\mathrm{L}$ & $7.34 \times 10^{-3}$ & $* 1.60 \times 10^{-3}$ & $2.42 \times 10^{-3}$ & $* 4.76 \times 10^{-5}$ & $1.08 \times 10^{-5}$ & $* 1.13 \times 10^{-7}$ \\
\hline
\end{tabular}


Fig. 7 Visual comparison of a one SISIM realization and $\mathbf{b}$ one HSISIM realization of MB2. The model block is discretized in $155 \times 115 \times 27$ cubic cells with 2 -cm sides (vertical exaggeration $2 \times$ ). Note that facies $L_{1 / 2}$ is not present in MB2. However, here the same colour scale used for MB3 in Fig. 9 is used to allow direct comparisons
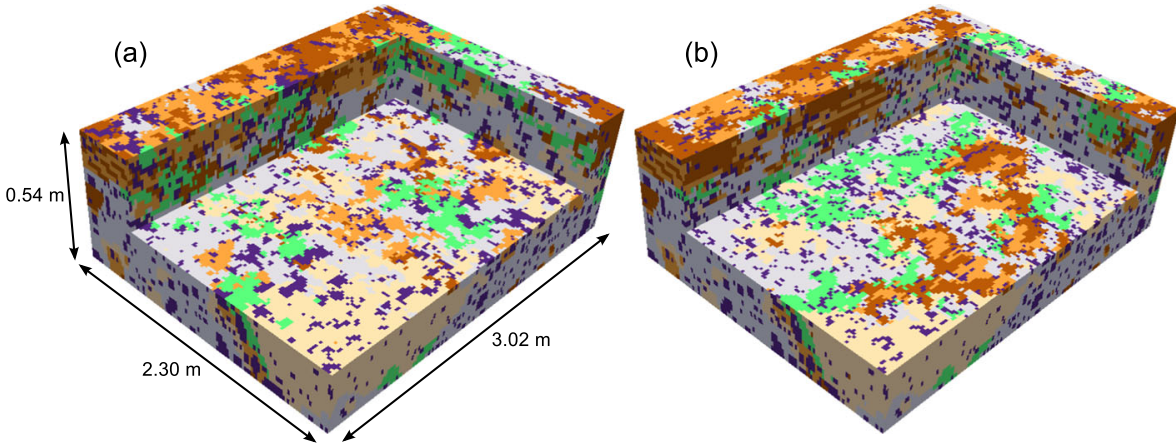

hierarchic technique, yielding a sufficiently good proxy of the real architecture.

The comparisons in terms of the reproduction of the target proportions and the connectivity indicators are made on the ensemble of realizations using a boxplot representation (Fig. 8): the minimum, the maximum, the 1 st and 3 rd quartiles and the median value of the considered quantity are shown; when a reference value is available, it is drawn as an horizontal green line. Overall, there are not big differences in the facies proportions obtained with SISIM and HSISIM, apart from a bigger spreading observed for the SISIM simulations and the better fit of HSISIM for the facies L (Fig. 8, top row). The greater spreading observed for SISIM in terms of facies proportions can also be observed for the total connectivity (Fig. 8, mid row). In addition, there is a difference of about two orders of magnitude between the total connectivity of facies L. In terms of intrinsic connectivity, the greater spreading observed for the other two variables for SISIM is less evident (Fig. 8, bottom row).

For the 3D simulations of MB3, the same criteria used for MB2 and illustrated in the previous section are adopted. MB3 is less structured and hence less anisotropic than MB2 (Fig. 9). It is therefore even more difficult to note clear differences between the two realizations. Despite this, in the HSISIM realization, a greater continuity in the well-sorted sandy gravel bodies (fSG) is apparent, and pebbles and cobbles are reproduced with a spatial distribution closer to the reference outcrops.

As in the case of MB2, this is an important feature of the HSISIM simulations that capture the contrast between the homogeneous sandy gravel framework with randomly scattered pebbles (SG, G, L) and the elongated sandy lenses (fSG, cS and fS).
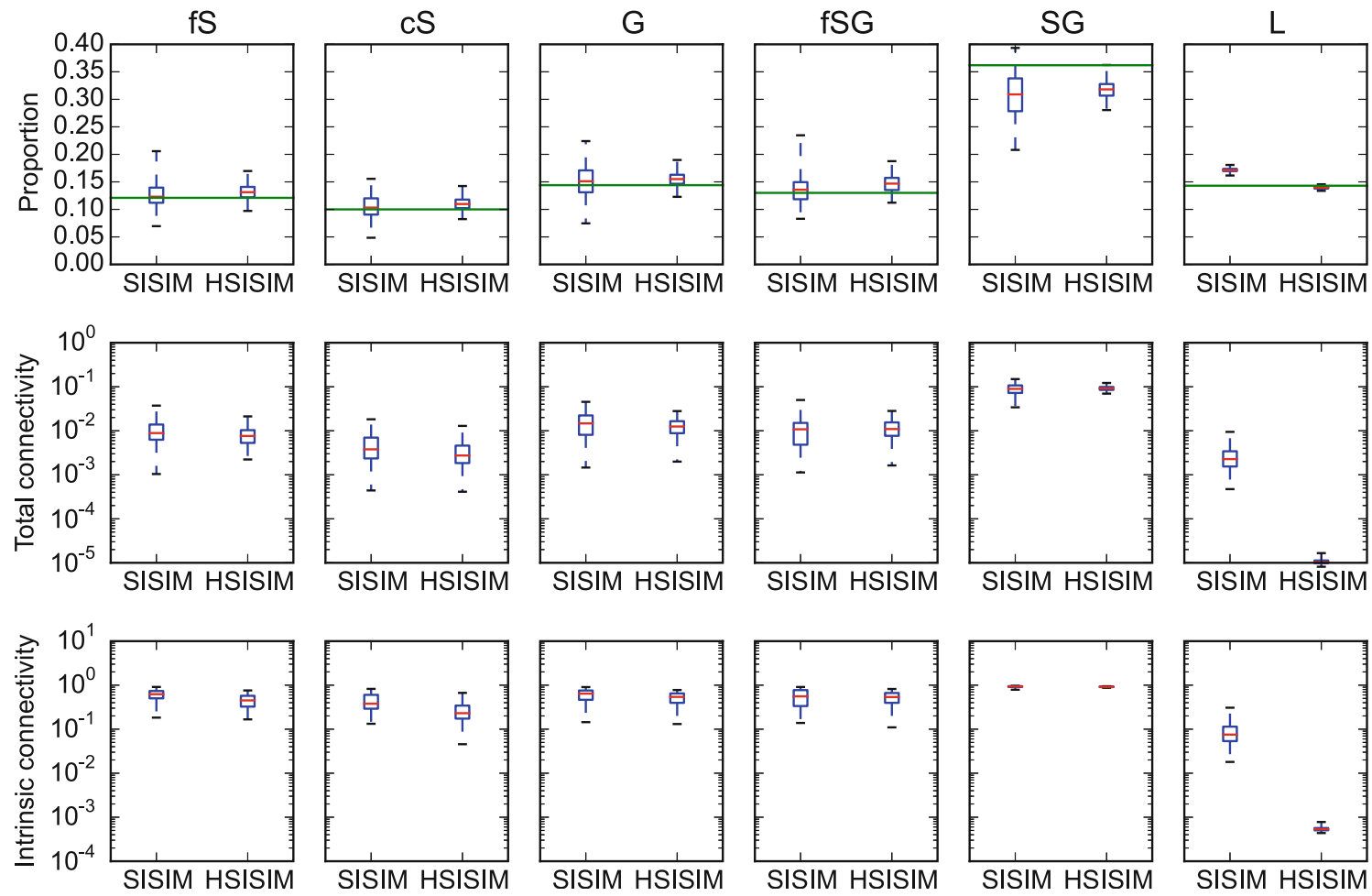

Fig. 8 Boxplots of proportions, total and intrinsic connectivity obtained with SISIM and HSISIM for 100 realizations of MB2. The green horizontal line represents the facies proportions of the mapped data set 
Fig. 9 Visual comparison of a one SISIM realization and $\mathbf{b}$ one HSISIM realization of MB3. The model block is discretized in $175 \times 122 \times 30$ cubic cells with 2 -cm sides (vertical exaggeration of $2 \times$ )
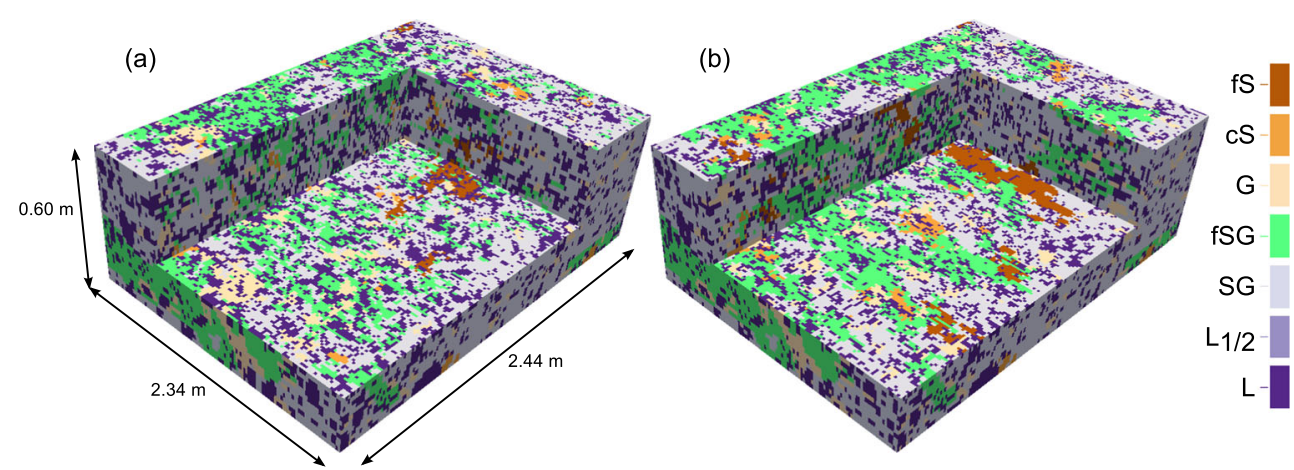

Both SISIM and HSISIM reproduce the target facies proportions relatively well (Fig. 10, top row). For both SISIM and HSISIM, the target proportions are included in the range of variability within the realizations for five out of seven of the facies, but the median values of the HSISIM proportions for $\mathrm{L}$ and $\mathrm{L}_{1 / 2}$ are closer to the reference. While the total and intrinsic connectivity indicators have a range of variability of five orders of magnitude within the different facies, overall the values obtained with the two techniques are comparable (Fig. 10, mid and bottom rows). For facies $\mathrm{L}_{1 / 2}$, only the differences are about two orders of magnitude.

\section{Particle-tracking simulations}

A further comparison between SISIM and HSISIM is performed in terms of cumulative BTCs obtained from particletracking simulations (Fig. 11). The greater variability of the SISIM simulation in terms of proportions and to some extent in terms of connectivity indicators results in a greater variability in the cumulative BTCs. This is apparent not only by comparing the BTCs for the whole set of realizations (Fig. 11a for SISIM and b for HSISIM), but also by looking at the "interquartile band", i.e., the region of the BTC plot delimited by the first and the third quartile for each ensemble at each time (Fig. 11c). From the latter picture, three aspects which are related to the higher variability of SISIM with respect to HSISIM are very evident: (1) first arrivals for SISIM anticipate those for HSISIM; (2) a long tail is visible for SISIM, when $t>1,000 \mathrm{~s}$; (3) the interquartile band is greater for SISIM than for HSISM.

\section{Sensitivity analysis on HSISIM}

In the following, the sensitivity of the proposed hierarchical procedure to the simulation settings and the binary tree is investigated by first analyzing and comparing the statistics
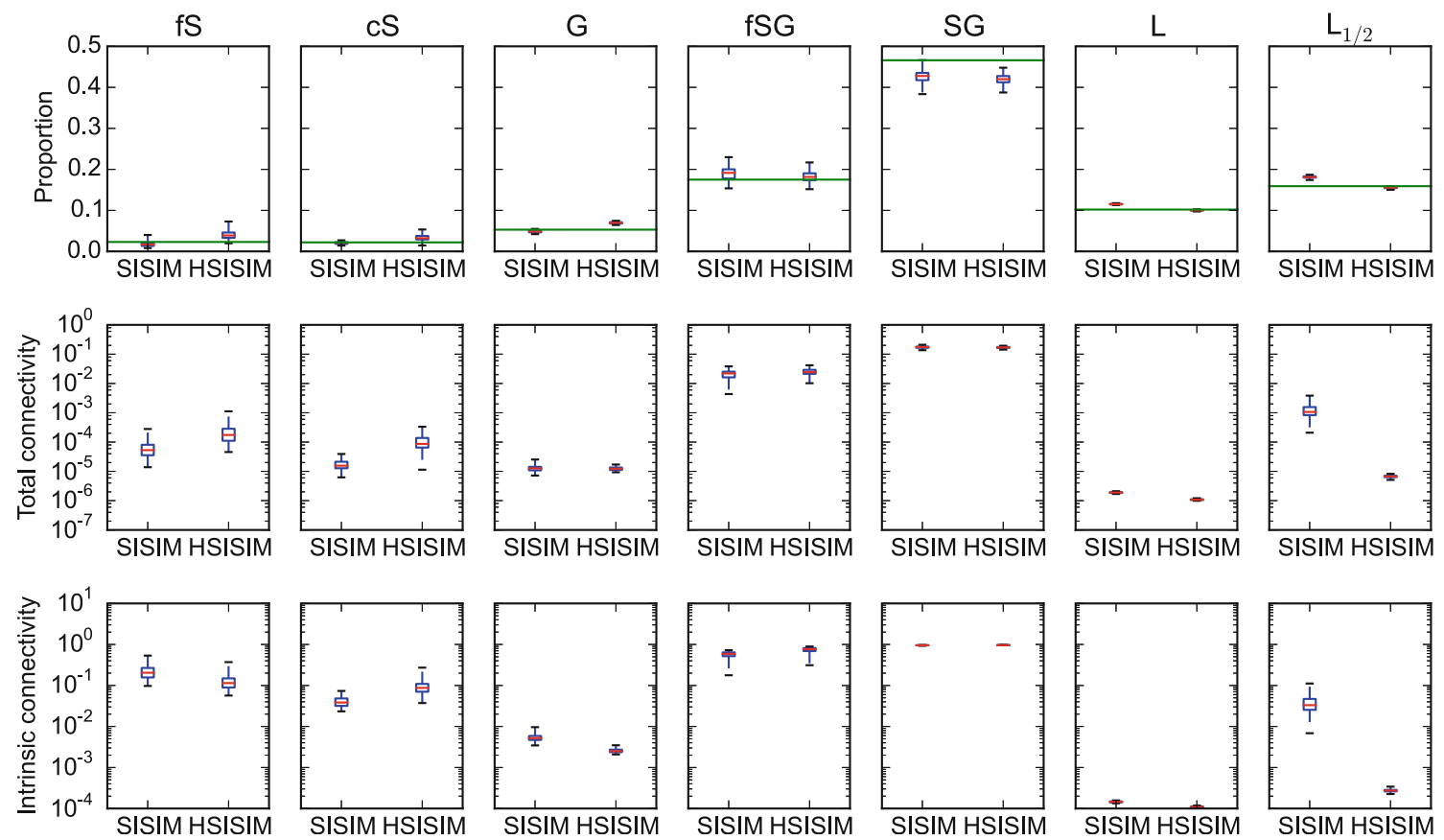

Fig. 10 Boxplots of proportions, total and intrinsic connectivity obtained with SISIM and HSISIM for MB3. The green horizontal line represents the facies proportions of the mapped data set 
(a) SISIM

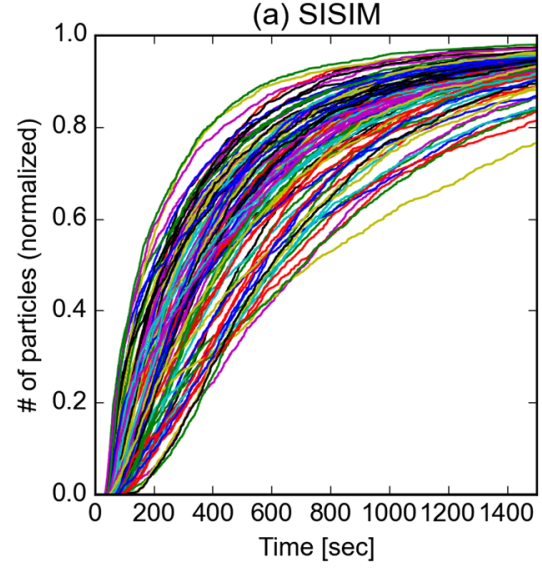

Fig. 11 Cumulative breakthrough curves obtained in the 3D particle tracking simulations performed for 100 SISIM and HSISIM realizations for MB2. a-b BTCs of each realization are drawn; $\mathbf{c}$ coloured areas are delimited by the first and third quartiles of the ensembles obtained with (b) HSISIM

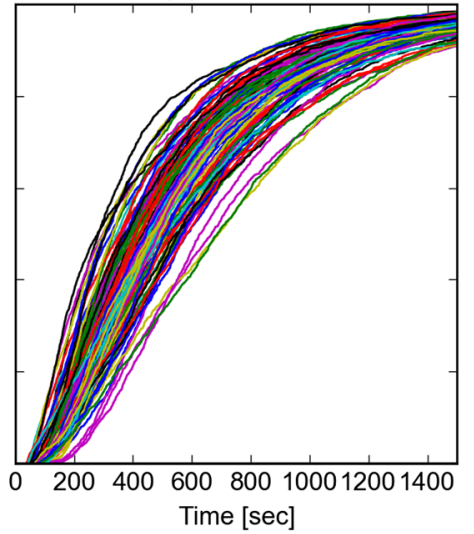

(c) SISIM and HSISIM

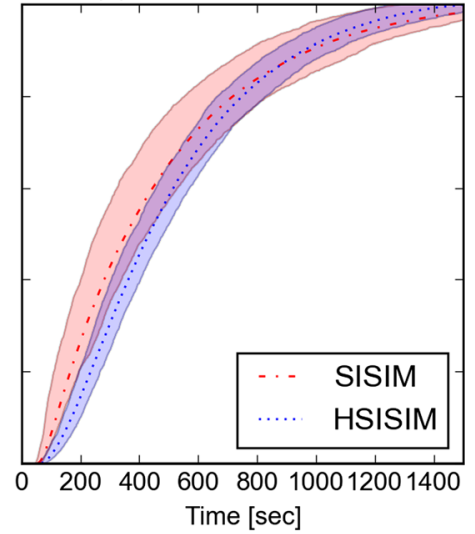

SISIM and HSISIM and the dotted lines join the median of each ensemble. The number of particles is normalized to allow a direct comparison

of $D_{1}$ and $v$ computed for the two blocks MB2 and MB3. Then, these same statistics are used, only for MB2, to investigate the sensitivity of the procedure to different simulation settings and different binary trees by means of transport simulations, performed with the KD approach on about $100 \mathrm{~m}$ long 1D domains.

\section{Statistical distributions of $D_{l}$ and $v$}

The particle-tracking simulations performed on each realization and illustrated in the previous sections served an additional purpose: to compute statistical distributions of $D_{1}$ and $v$ to be used in the KD approach. Although some BTCs could be better approximated with bi-modal curves (Fig. 12), here the 100 BTCs obtained for the HSISIM realizations are considered mono-modal and fitted with Eq. (4) to obtain statistical distribution for $D_{1}$ and $v$.

Table 4 summarizes the statistics for $D_{1}$ and $v$ respectively, reporting the 5th percentile and 95th percentile of the distributions used in the Monte Carlo simulation. In addition, the tables report the mean and the median values as well as the parameters $(\mu$ and $\sigma)$ of Gaussian distributions fitted on the logarithm of the variables $D_{1}$ and $v$. These same statistics are represented in terms of histograms in Fig. 13. In MB2, the presence of a strong anisotropy characterized by horizontal layers with high hydraulic conductivity makes the value of $D_{1}$ and $v$ greater than one order of magnitude for this model block than for MB3.

\section{Kolmogorov-Dmitriev approach on extended $1 D$ domains}

Using the statistical distribution of $D_{1}$ and $v$ illustrated in the previous section, 1D transport simulations can be performed with the KD approach on simulation domains two orders of magnitude larger than the model blocks. While the representative scale length of $D_{1}$ and $v$ is the same for both the $3 \mathrm{D}$ particle tracking and the $1 \mathrm{D} \mathrm{KD}$ simulations, the $\mathrm{KD}$ approach is computationally more efficient and allows for simulation on more extended domains.

In the previous sections, only the results of the hierarchical simulations performed using simple Kriging (SK) and a textural hierarchy are reported. Figure 14, instead, contains a comparison of the BTCs obtained with other simulation settings with the purpose of illustrating the flexibility provided by the proposed hierarchical procedure. The simulations settings considered are: (1) a hierarchy based on hydrofacies abundance and SK at every node of the binary tree; (2) a hierarchy based on hydrofacies abundance and SK or ordinary Kriging (OK) applied in different simulation nodes; (3) a textural hierarchy and SK at every node of the binary tree; (4) a textural hierarchy and SK or OK applied in different simulation nodes.

Given the same hierarchy, the particles travel faster in the domains simulated using SK at every node and, for these domains, there is a good fit with the analytical distribution computed using the median values of $D_{1}$ and $v$ (black dashed lines, Fig. 14). Instead, for the simulations performed using alternatively SK and OK, the peak of particles are more intense but reach the control planes hundreds of seconds after the analytical BTCs (Fig. 14). In addition, the more pronounced peaks of the KD simulations using alternatively SK and $\mathrm{OK}$ correspond to a tail concentration that decreases faster than the analytical BTCs.

\section{Discussion and conclusions}

This work presents a thorough exploration of a hierarchical simulation procedure, that here is validated and compared with a standard geostatistical simulation tool, SISIM. The 

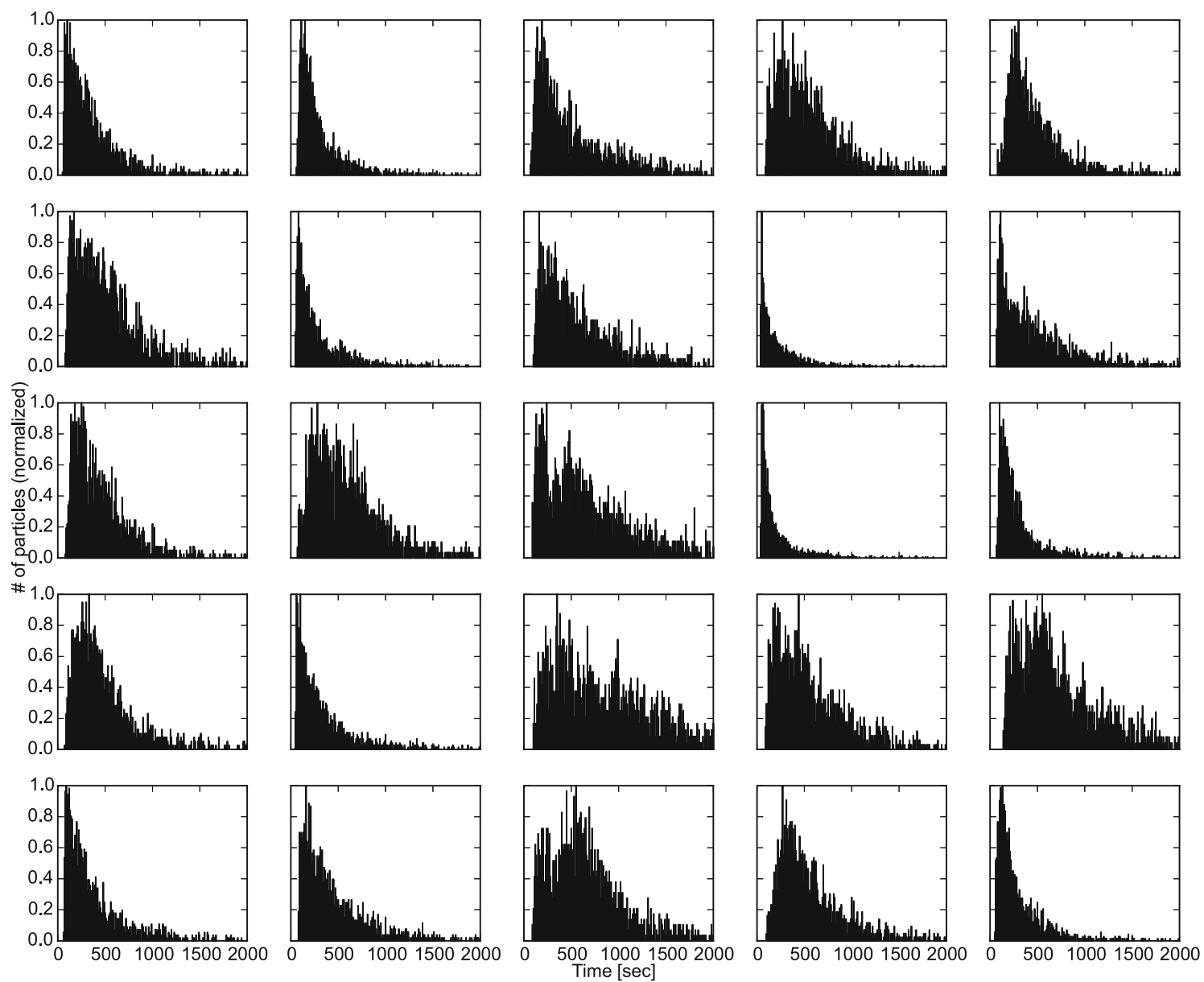

Fig. 12 Particle-tracking BTCs for the first 25 HSISIM simulations of MB2. Note how some curves are far from unimodal distributions

comparisons are first performed on the reproduction of 2D domains, where reference outcrops are available and can be used to evaluate the reliability of the proposed simulation procedure. When using the hierarchical simulation procedure, the reproduced heterogeneity appears closer to the reference outcrops, both in terms of connectivity indicators and in terms of facies proportions. In addition, the considered reference outcrops, that represent the mapped surface of two model blocks of fluvio-glacial sediments, are used to reconstruct the heterogeneity of the blocks in 3D with the hierarchical procedure and with SISIM. The 3D simulation does not allow a direct comparison with a true reference value, but some differences can be noticed between the connectivity indicators and the facies proportions computed on ensembles of 100 simulations for each simulation method. Particle-tracking simulations performed on the ensembles serves to illuminate noticeable differences in terms of transport behavior, which present a smaller variability for the HSISIM simulations. This smaller variability could be explained by the additional constraints included in the hierarchical procedure.

The results obtained from the particle-tracking experiments are used to expand the analysis of the transport behavior of the proposed hierarchical method using the KD approach, which allowed simulation on domains with a length two orders of magnitude greater than the sides of the blocks.
Table 4 Statistics for $D_{l}$ and $v$ obtained with particle tracking on 100 simulations for HSISIM. A Gaussian distribution with parameters $\mu$ and $\sigma$ is fitted to the $\log$ transformed variable $D_{l}$ and $v$

\begin{tabular}{lccllll}
\hline Block & 5th percentile & 95th percentile & Median & Mean & $\mu$ & $\sigma$ \\
\hline \multicolumn{2}{l}{ Longitudinal dispersion coefficient $D_{1}\left[\mathrm{~m}^{2} \mathrm{~s}^{-1}\right]$} & & & & \\
MB2 & $2.80 \times 10^{-3}$ & $8.29 \times 10^{-3}$ & $4.82 \times 10^{-3}$ & $5.09 \times 10^{-3}$ & -5.34 & $3.30 \times 10^{-1}$ \\
MB3 & $2.46 \times 10^{-4}$ & $3.57 \times 10^{-4}$ & $2.96 \times 10^{-4}$ & $2.98 \times 10^{-4}$ & -8.13 & $1.13 \times 10^{-1}$ \\
Average pore-water velocity $v\left[\mathrm{~m} \mathrm{~s}^{-1}\right]$ & & & & \\
MB2 & $4.03 \times 10^{-3}$ & $6.68 \times 10^{-3}$ & $5.19 \times 10^{-3}$ & $5.25 \times 10^{-3}$ & -5.26 & $1.54 \times 10^{-1}$ \\
MB3 & $1.53 \times 10^{-3}$ & $1.75 \times 10^{-3}$ & $1.63 \times 10^{-3}$ & $1.63 \times 10^{-3}$ & -6.42 & $4.08 \times 10^{-2}$ \\
\hline
\end{tabular}


(a)

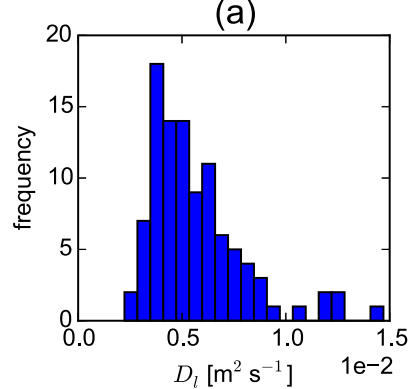

(c)

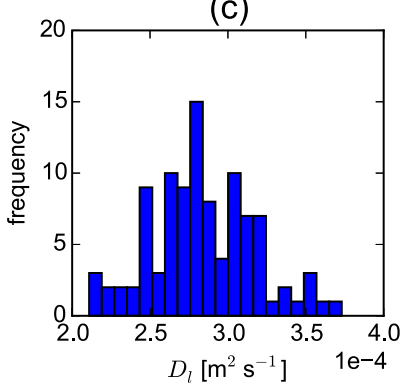

(b)

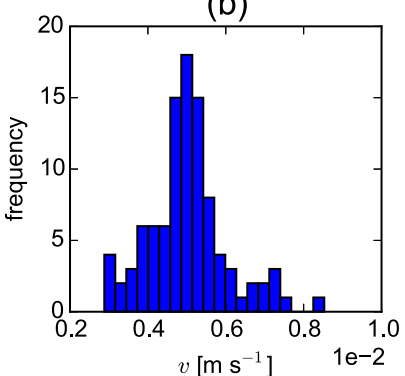

(d)

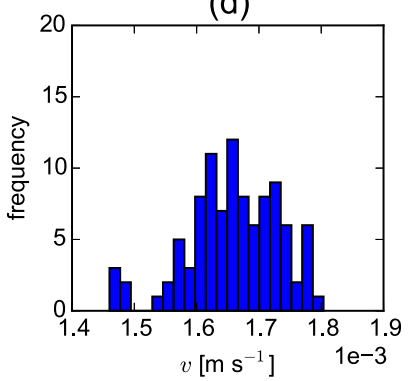

Fig. 13 Histograms of the results of the particle tracking performed on the 100 realizations obtained with HSISIM: a $D_{1}$ for MB2; $\mathbf{b} v$ for MB2; c $D_{1}$ for MB3; $\mathbf{d} v$ for MB3

A fundamental aspect of the proposed hierarchical simulation procedure is its flexibility, which can be achieved by changing the simulation settings at two levels. At the node level, one can play with the simulation settings offered by the selected simulation method. In this work, the possibility of changing the Kriging strategy offered by SISIM (Deutsch and Journel 1997) is tested using ordinary Kriging and simple Kriging at different nodes of the simulation binary tree (Fig. 14). In addition, the hierarchical approach allows for the use of a different variogram model for sediments classified with the same hydrofacies code but belonging to a different sub-set (node) in a straightforward manner. Other techniques would have required an additional zonation of the simulation domain. When using another simulation engine for the node level simulations, one could have at his disposal different simulation settings. At the binary tree level, the potential to select a different binary tree provides great flexibility as well as the possibility to include additional geological knowledge in the simulation procedure. In this study, the focus is on the results obtained with a binary tree based on the textural hierarchy proposed by Zappa et al. (2006). In addition, Fig. 14 presents results obtained with a binary tree based on facies abundance; note, however, that other hierarchical simulation binary trees
Fig. 14 BTCs obtained with the $\mathrm{KD}$ approach on domains with an extension of about $100 \mathrm{~m}$ using the statistics of MB2. Each part of the figure corresponds to a different simulation setting. The control planes are located at distances $7.72 \mathrm{~m}$ (blue lines), $25.48 \mathrm{~m}$ (green lines), $42.48 \mathrm{~m}$ (red lines), $59.48 \mathrm{~m}$ (cyan lines) and $73.98 \mathrm{~m}$ (fuchsia lines) from the injection plane. The black dashed lines are the analytical distributions computed using the median values of $D_{1}$ and $v$
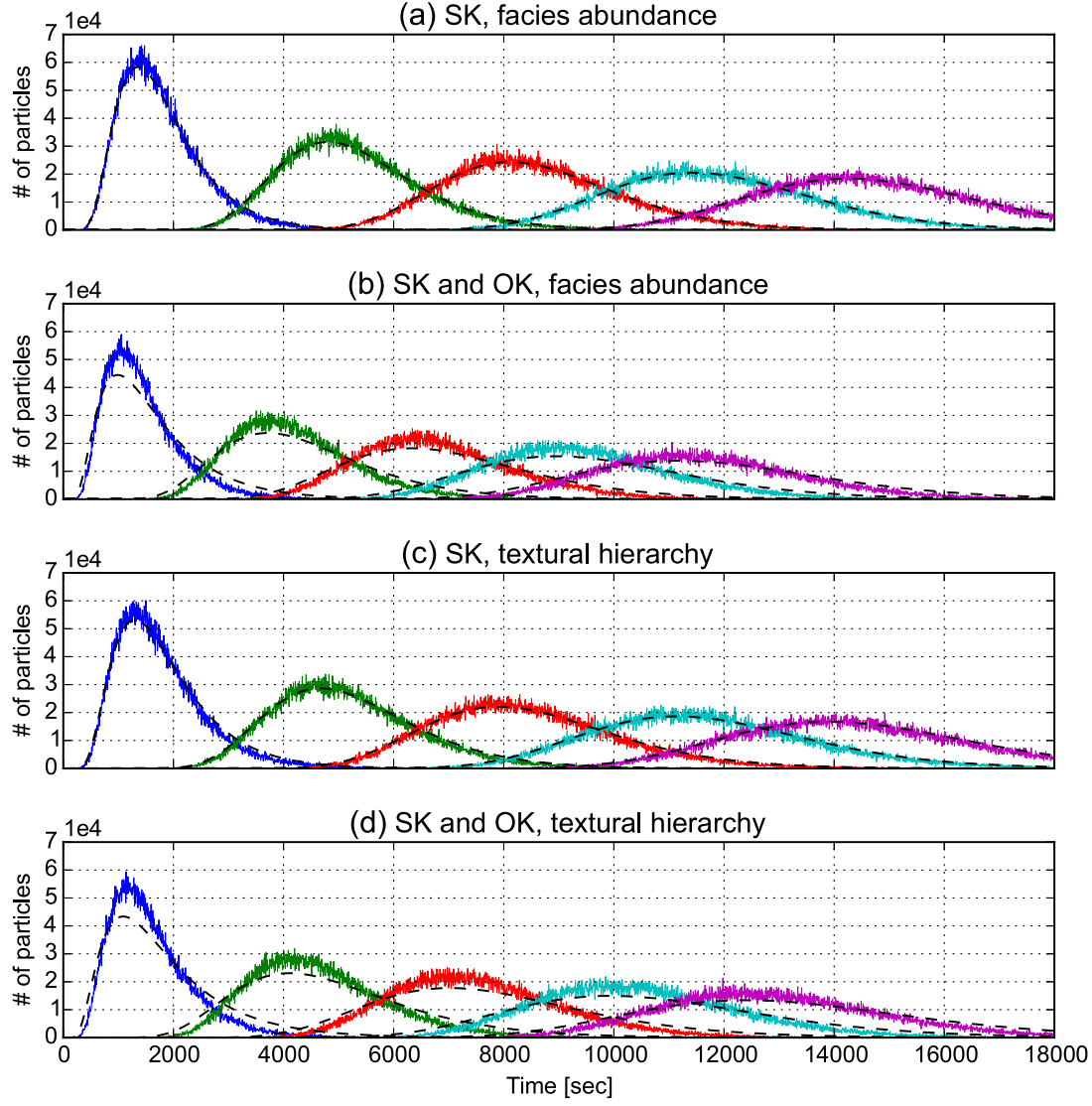
could be selected, for example a binary tree based on grainsize distribution, or more complex binary trees extracted from relational databases where the sedimentary architecture is represented by means of genetic units, corresponding to different scales and nested in a hierarchical manner (Colombera et al. 2012). Therefore, although the definition of the binary tree may require additional effort, very often, similar effort is required to carefully apply any other geostatistical simulation technique, and the required information can be borrowed from similar case studies. The flexibility available when changing the simulation setting both at node and at binary tree level is illustrated by the differences between the BTCs computed with the KD approach applied on domains with lengths of about $100 \mathrm{~m}$ (Fig. 14).

In this study, the variographic analysis is deliberately kept at a simple level, ignoring the trends and the non stationarities of the data-set and considering only different model parameters along the vertical and the horizontal directions. However, the proposed hierarchical simulation procedure allows for partial surpassing of the limitations of a simple variographic analysis. Overall, the procedure is more robust and easier to verify, because binary simulations can be easily managed, modeled with a variogram, and checked either visually or in a quantitative way, e.g., with connectivity indicators.

The hierarchical simulation procedure proposed in this work represents an alternative to the more complex hierarchical frameworks that require the blending of two or more interpolation and simulation techniques. The proposed hierarchical procedure implemented here is based on SISIM and called HSISIM. Being based on SISIM, the proposed procedure still suffers from some of SISIM's limitations (Emery 2004), namely the difficulty in giving a clear geological meaning to the range of the indicator variograms, together with the limitations in reproducing the connectivity of complex geological patterns, shared by other variogram-based techniques (Schlüter and Vogel 2011; Western et al. 2001; Zinn and Harvey 2003). However, the hierarchical simulation procedure itself has general validity and could be applied to other facies simulation algorithms, including multiple-point statistics, transition-probability Markov chain, and truncated pluriGaussian-based approaches. In fact, it is expected that this hierarchical procedure can be applied to situations characterized by three or more facies and implemented using any method which permits performing (at least) binary simulations.

The KD approach turned out to be a valuable tool to efficiently investigate the transport properties of the considered heterogeneities on domains extending up to $100 \mathrm{~m}$ of length. In order to expand the results on the case studies considered in this paper, further studies are required to fully exploit its flexibility, for example to include the transport of reactive contaminants.

\section{References}

Anderson MP (1989) Hydrogeological facies models to delineate largescale spatial trends in glacial and glaciofluvial sediments. Geol Soc Am Bull 101(4):501-511. doi:10.1130/0016-7606(1989) $101<0501$ :HFMTDL $>2.3$. CO;2

Anderson MP, Woessner WW (1991) Applied groundwater modeling: simulation of flow and advective transport. Academic, San Diego, CA

Baratelli F, Giudici M, Vassena C (2011) Single and dual-domain models to evaluate the effects of preferential flow paths in alluvial sedi-ments. Trans Por Med 87(2):465-484. doi:10.1007/ s11242-010-9695-4

Baratelli F, Giudici M, Parravicini G (2014) Single- and dualdomain models of solute transport in alluvial sediments: the effects of het-erogeneity structure and spatial scale. Trans Por Med 105(2):315348. doi:10.1007/s11242-014-0371-y

Bersezio R, Bussolini C, Pagani F, Viviani C (1999) The Pleistocene fluvio-glacial sediments of the Lower Ticino Valley: stratigraphy, sedimentary features and relations with the hydrogeological properties at a gravel pit, south of Lake Verbano. Geol Insubrica 4:49-60

Carle S (1996) A transition probability-based approach to geostatistical characterization of hydrostratigraphic architecture. PhD Thesis, University of California Davis, USA

Cherubini C, Musci F, Pastore N (2009) Stochastic geolithological reconstruction coupled with artificial neural networks approach for hydrogeological modeling. Int J Math Model Method Appl Sci 3(2):105-115

Colombera L, Felletti F, Mountney NP, McCaffrey WD (2012) A database approach for constraining stochastic simulations of the sedi-mentary heterogeneity of fluvial reservoirs. AAPG Bull 96(11): 2143-2166. doi:10.1306/04211211179

Comunian A, Renard P, Straubhaar J, Bayer P (2011) Three-dimensional high resolution fluvio-glacial aquifer analog, part 2: geostatistical modeling. J Hydrol 405(1-2):10-23. doi:10.1016/ j.jhydrol.2011. 03.037

Dell'Arciprete D, Vassena C, Baratelli F, Giudici M, Bersezio R, Felletti F (2014) Connectivity and single/dual domain transport models: tests on a point-bar/channel aquifer analogue. Hydrogeol J 22(4):761778. doi:10.1007/s10040-014-1105-5

Deutsch CV, Journel AG (1997) GSLIB: geostatistical software library and user's guide, 2nd edn. Oxford University Press, Oxford, UK Emery X (2004) Properties and limitations of sequential indicator simulation. Stoch Environ Res Risk Assess 18:414-424.

doi:10.1007/s00477-004-0213-5

Giacobbo F, Patelli E (2007) Monte Carlo simulation of nonlinear

reactive contaminant transport in unsaturated porous media. Ann Nucl Energ 34(1-2):51-63. doi:10.1016/j.anucene.2006.11.011

Giudici M, Bersezio R, Felletti F, Baratelli F, Cattaneo L, Cavalli E, Dell'Arciprete D, Mele M, Pessina L, Vassena C (2012) A multidisciplinary study of sediments' connectivity and transport parameters for aquifer analogues. In: Models: repositories of knowledge, Proceedings ModelCARE2011, Leipzig, Germany, September 2011, IAHS Publ. 355, IAHS, Wallingford, UK, pp 223-228

Guardiano FB, Srivastava RM (1993) Multivariate geostatistics: beyond bivariate moments. In: Soares A (ed) Geostatistics: Troia'92, vol 1. Kluwer, Dordrecht, The Netherlands, pp 133-144

Hubert J (1970) Effect of injection speed on the tracer concentration curves. Nukleonika 15:371-376

Isaaks EH, Srivastava RM (1989) An introduction to applied geostatistics. Oxford University Press, Oxford, UK

Jussel P, Stauffer F, Dracos T (1994) Transport modeling in heterogeneous aquifers: 1 . statistical description and numerical generation 
of gravel deposits. Water Resour Res 30(6):1803-1817. doi:10. 1029/94WR00162

Klingbeil R, Kleineidam S, Asprion U, Aigner T, Teutsch

G (1999) Relating lithofacies to hydrofacies: outcrop-based hydrogeological characterisation of Quaternary gravel deposits. Sedim Geol 129(3-4):299-310. doi:10.1016/ S0037-0738(99)00067-6

Kolmogorov AN, Dmitriev NA (1947) Branching stochastic processes. Dokl Akad Nauk SSSR 56:7-10

Kreft A, Zuber A (1978) On the physical meaning of the dispersion equation and its solutions for different initial and boundary condi-tions. Chem Eng Sci 33(11):1471-1480. doi:10.1016/0009-2509(78)85196-3

Le Loc'h G, Beucher H, Galli A, Doligez B (1994) Improvement in the truncated Gaussian method: combining several Gaussian functions. In: 4th European Conference on the Mathematics of Oil Recovery. Session: Heterogeneity description and assessment of uncertainty. http://www.earthdoc.org/ publication/publicationdetails/?publication $=49733$.

Accessed 24 March 2015

Marseguerra M, Zio E (1997) Modelling the transport of contaminants in groundwater as a branching stochastic process. Ann Nucl Energ 24(8):625-644. doi:10.1016/S0306-4549(96)00097-7

Renard P, Allard D (2013) Connectivity metrics for subsurface flow and transport. Adv Water Resour 51:168-196. doi:10.1016/ j.advwatres. 2011.12.001

Schlüter S, Vogel HJ (2011) On the reconstruction of structural and functional properties in random heterogeneous media. Adv Water Resour 34(2):314-325. doi:10.1016/j.advwatres.2010.12.004
Seifert D, Jensen J (1999) Using sequential indicator simulation as a tool in reservoir description: issues and uncertainties. Math Geol

31(5): 527-550. doi:10.1023/A:1007563907124

Strebelle S (2002) Conditional simulation of complex geological structures using multiple-point statistics. Math Geol 34:1-21. doi:10. 1023/A:1014009426274

Vassena C, Cattaneo L, Giudici M (2010) Assessment of the role of facies heterogeneity at the fine scale by numerical transport experiments and connectivity indicators. Hydrogeol $\mathrm{J}$ 18(3):651-668. doi:10. 1007/s10040-009-0523-2

Vukovic M, Soro A (1992) Determination of hydraulic conductivity of porous media from grain-size composition. Water Resources, Highlands Ranch CO, 86 pp

Weissmann G, Fogg G (1999) Multi-scale alluvial fan heterogeneity modeled with transition probability geostatistics in a sequence strat-igraphic framework. J Hydrol 226(1-2):48-65. doi:10.1016/ S0022-1694(99)00160-2

Western AW, Böschl G, Grayson RB (2001) Toward capturing

hydrologically significant connectivity in spatial patterns. Water

Resour Res 37(1):83-97. doi:10.1029/2000WR900241

Zappa G, Bersezio R, Felletti F, Giudici M (2006) Modeling heterogeneity of gravel-sand, braided stream, alluvial aquifers at the facies scale. J Hydrol 325(1-4):134-153. doi:10.1016/ j.jhydrol.2005.10. 016

Zinn B, Harvey C (2003) When good statistical models of aquifer het-erogeneity go bad: a comparison of flow, dispersion, and mass transfer in connected and multivariate Gaussian hydraulic conductivity fields. Water Resour Res 39(3):1051. doi:10.1029/2001WR001146 